\title{
Gene Therapy for Osteoarthritis Treatment and Joint Preservation
}

\author{
Liang Gao ${ }^{1,2}$ \\ ${ }^{1}$ Center of Experimental Orthopaedics, Saarland University Medical \\ Center, Homburg, Saarland, Germany \\ ${ }^{2}$ Sino Euro Orthopaedics Network, Homburg, Saarland, Germany \\ Int J Recent Surg Med Sci 2019;5:2-3
}

Osteoarthritis (OA) affects the entire joint structures, which are unable to be completely regenerated with current clinical treatments. ${ }^{1}$ Even the concept of OA gene therapy has been proposed over the past two decades, only five clinical trials have ever been undertaken. ${ }^{2}$ Recent translational advances of OA gene therapy highlight noteworthy therapeutic benefits with excellent safety records.

Current vector-based gene delivery uses both nonviral and viral vectors. ${ }^{3}$ The nonviral vectors carrying modified therapeutic DNAs are introduced into cells via various procedures, and viral vectors are deliberately engineered and can naturally infect mammalian cells. Both types of vectors can deliver genes either into isolated cells (ex vivo) initially and transferred subsequently into human body or into targeted locations of human body (in vivo) directly. Intra-articular gene therapy with non-viral vectors often has an evanescent effect due to the physiological self-cleaning within human body, which is generally inappropriate for chronic diseases such as osteoarthritis. Viral vectors with therapeutic genes can be delivered and resided intracellularly, allowing for endogenous synthetization of therapeutic products, and obtaining durable gene expressions.

Ex vivo genetic modification has been preclinically demonstrated in cellular, tissue, and animal models with various viral vectors. ${ }^{4}$ Ex vivo approaches with autologous cells are disadvantaged with multiple operations and consecutively high costs, which can be overcome by the utilization of allogeneic cells or in vivo gene delivery. ${ }^{5}$ The utilization of allogeneic cells avoids the individual harvesting of autologous cells from patients. In vivo gene delivery by direct intra-articular injection is an alternative to accelerate OA treatment; however, choosing appropriate vectors is crucial for further translations due to the diverse immunogenic, inflammatory, and hazardous characteristics of various vectors. Recombinant adenoassociated virus ( $\mathrm{rAAV}$ ) has emerged as a promising candidate for both ex vivo and in vivo gene therapy with advantages of nonpathogenicity, reduced immunogenicity, and extended transgene expression duration compared with other vectors. ${ }^{6}$ Particularly, the main remarkability of rAAV-based in vivo gene therapy is the possibility of durable clinical benefits following a simple well-tolerated intra-articular injection as well as the ease of manufacturing a formulated therapeutic product, rather than a personalized product in ex vivo gene therapy, which might further assist its rapid commercial distribution.

Recent breakthroughs from clinical trials mark the beginning of a fascinating era of genetic medicine. On July 12, 2017, the South Korean Ministry of Food and Drug Safety approved the world's first gene therapy (Invossa, TissueGene-C) for the treatment of knee OA (Kellgren-Lawrence grade 3), which was based on allogeneic chondrocytes ex vivo transduced with a retrovirus encoding transforming growth factor- $\beta 1{ }^{7}$ Since July 2018, the pivotal phase III trials for U.S. approval of invossa has been initiated with 1,020 participants at over 50 clinics across the United States. ${ }^{8}$ Meanwhile, since May 2019, another phase I trial was recruiting for 9 participants in the United States to test the safety and effectiveness of in vivo intra-articular injection of rAAV-mediated human interleukin-1 receptor antagonist transgene (rAAV-IL-1Ra) for knee OA treatment. ${ }^{9}$ However, no clinical trial currently exists for gene therapy of focal cartilage defects, which potentially initiate a general osteoarthritis.

More excitingly, the emerging clustered regularly interspaced short palindromic repeats (CRISPR)/Cas9based geneediting technology offers a powerful tool for the efficient and targeted editing of OA-related genes. ${ }^{10}$ Such a region-specific editing of the genome evades the risk of insertional mutagenesis and uncertainly regulated transgeneexpression in traditional vector-based gene deliveries. Various studies have been undertaken toward treatments of multiple diseases (e.g., human immunodeficiency virus [HIV], ${ }^{11} \beta$-thalassaemia, ${ }^{12}$ and sickle-cell disease ${ }^{13}$ ) through either the nonhomologous end joining or homology-directed repair pathway. However, several hurdles are necessitated to overcome before this technique can be adequately prepared for further clinical translation. First, the cell response to DNA damages varies to cell type and status, and the underlying mechanism of repair
(C2019 Medical and Surgical Update Society
License terms

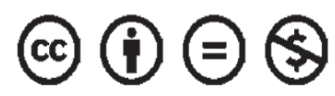

DOI https://doi.org/

10.1055/s-0039-1692612

Liang Gao, MD, PhD, Center of ISSN 2455-7420. 
pathway choice is poorly understood. Second, for ex vivo gene editing, safe and feasible clinical translation are practically difficult, when the postediting products rely requisitely on extensive ex vivo amplification of edited cell clones. Third, comprehensive assessment of the offtarget activity of the selected nuclease and cellular immunogenicity is critical, especially if the delivered genes are expressed over the long term.

In summary, the up-to-date gene therapy strategies enable a targeted delivery of information-rich therapeutic genes and region-specific editing of OA-related genes, allowing for a stable, durable, and controlled therapeutic gene expression for OA treatment. Future rapid progresses and endeavors of gene therapy in a translational framework are expected to alleviate OA patients' sufferings with early interventions toward the joint preservation.

\section{Conflict of Interest}

None declared.

\section{References}

1 Wieland HA, Michaelis M, Kirschbaum BJ, Rudolphi KA. Osteoarthritis - an untreatable disease? Nat Rev Drug Discov 2005;4(4):331-344

2 Evans CH, Ghivizzani SC, Robbins PD. Arthritis gene therapy is becoming a reality. Nat Rev Rheumatol 2018;14(7):381-382

3 Evans $\mathrm{CH}$, Huard J. Gene therapy approaches to regenerating the musculoskeletal system. Nat Rev Rheumatol 2015;11(4): 234-242
4 Cucchiarini M, Madry $H$. Biomaterial-guided delivery of gene vectors for targeted articular cartilage repair. Nat Rev Rheumatol 2019;15(1):18-29

5 Wang D, Gao G. State-of-the-art human gene therapy: part I. Gene delivery technologies. Discov Med 2014;18(97):67-77

6 Rey-Rico A, Cucchiarini M. smart and controllable rAAV gene delivery carriers in progenitor cells for human musculoskeletal regenerative medicine with a focus on the articular cartilage. Curr Gene Ther 2017;17(2):127-138

7 Kim MK, Ha CW, In Y, et al. A multicenter, double-blind, phase III clinical trial to evaluate the efficacy and safety of a cell and gene therapy in knee osteoarthritis patients. Hum Gene Ther Clin Dev 2018;29(1):48-59

8 Cherian JJ, Parvizi J, Bramlet D, Lee KH, Romness DW, Mont MA. Preliminary results of a phase II randomized study to determine the efficacy and safety of genetically engineered allogeneic human chondrocytes expressing TGF- $\beta 1$ in patients with grade 3 chronic degenerative joint disease of the knee. Osteoarthritis Cartilage 2015;23(12):2109-2118

9 Evans CH, Ghivizzani SC, Robbins PD. Arthritis gene therapy approved in Korea.J Am Acad Orthop Surg 2018;26(2):e36-e38

10 Almarza D, Cucchiarini M, Loughlin J. Genome editing for human osteoarthritis-a perspective. Osteoarthritis Cartilage 2017;25(8):1195-1198

11 Liao HK, Gu Y, Diaz A, et al. Use of the CRISPR/Cas9 system as an intracellular defense against HIV-1 infection in human cells. Nat Commun 2015;6:6413

12 Bank A, Dorazio R, Leboulch P. A phase I/II clinical trial of beta-globin gene therapy for beta-thalassemia. Ann N Y Acad Sci 2005; 1054:308-316

13 Hoban MD, Lumaquin D, Kuo CY, et al. CRISPR/Cas9-Mediated correction of the sickle mutation in human CD34+ cells. Mol Ther 2016;24(9):1561-1569 